\title{
Ruin Probabilities with a Markov Chain Interest Model
}

\author{
by \\ Jun Cai \\ University of Waterloo \\ and \\ David C M Dickson \\ The University of Melbourne \\ RESEARCH PAPER NUMBER 101
}

August 2002

Centre for Actuarial Studies

Department of Economics

The University of Melbourne

Victoria 3010

Australia 


\title{
Ruin Probabilities with a Markov Chain Interest Model
}

\author{
Jun Cai ${ }^{a}$ and David C. M. Dickson ${ }^{b}$ \\ ${ }^{a}$ Department of Statistics and Actuarial Science \\ University of Waterloo \\ Waterloo, Ontario N2L 3G1 \\ Canada \\ Email: jcai@math.uwaterloo.ca \\ and \\ ${ }^{b}$ Centre for Actuarial Studies \\ Faculty of Economics and Commerce \\ University of Melbourne \\ Victoria 3010 \\ Australia \\ Email: dcmd@unimelb.edu.au
}

\begin{abstract}
Ruin probabilities in two generalized discrete time risk processes with a Markov chain interest model are studied. Recursive and integral equations for the ruin probabilities are given. Generalized Lundberg inequalities for the ruin probabilities are derived both by inductive and martingale approaches. The relationships between these inequalities are discussed. A numerical example is given to illustrate these results.
\end{abstract}

Keywords: Risk process; Markov chain; ruin probability; rate of interest; Lundberg's inequality; Jensen's inequality; NWUC; supermartingale; optional stopping theorem.

AMS 1991 SUBJECT CLASSIFICATION: PRIMARY 60K10; 60K25

SECONDARY $62 \mathrm{~N} 05$ 


\section{Introduction}

Let $\left\{X_{n}, n=1,2, \cdots\right\}$ and $\left\{Y_{n}, n=1,2, \cdots\right\}$ be two sequences of independent and identically distributed (i.i.d.) non-negative random variables. They have common distribution functions $H(x)=\operatorname{Pr}\left\{X_{1} \leq x\right\}$ and $F(y)=\operatorname{Pr}\left\{Y_{1} \leq y\right\}$, respectively, with $F(0)=0$. Define

$$
\psi(u)=\operatorname{Pr}\left\{\bigcup_{k=1}^{\infty}\left(U_{k}<0\right)\right\}
$$

where

$$
U_{k}=u+\sum_{t=1}^{k}\left(X_{t}-Y_{t}\right), k=1,2, \cdots,
$$

or, equivalently, the stochastic process $\left\{U_{k}, k=1,2, \cdots\right\}$ satisfies

$$
U_{k}=U_{k-1}+X_{k}-Y_{k}, k=1,2, \cdots,
$$

with $U_{0}=u \geq 0$. Then $\psi(u)$ is the ultimate ruin probability in the classical risk model (1.1).

Let $\left\{I_{n}, n=0,1,2, \cdots\right\}$ be another sequence of non-negative random variables. Cai (2002a, 2002b) has considered two generalized discrete time risk processes by defining

$$
U_{k}=\left(U_{k-1}+X_{k}\right)\left(1+I_{k}\right)-Y_{k}, k=1,2, \cdots
$$

and

$$
U_{k}=U_{k-1}\left(1+I_{k}\right)+X_{k}-Y_{k}, k=1,2, \cdots
$$

respectively, with initial surplus $U_{0}=u$.

Mathematically, (1.2) and (1.3) are generalizations of the classical risk model (1.1). Practically, models (1.2) and (1.3) can be used to include the effect of timing of payments and interest on a surplus process as explained in Cai (2002a, 2002b). We study these effects by considering ruin probabilities.

It has been shown in Cai (2002a) that (1.2) implies

$$
U_{k}=u \prod_{j=1}^{k}\left(1+I_{j}\right)+\sum_{j=1}^{k}\left(\left(X_{j}\left(1+I_{j}\right)-Y_{j}\right) \prod_{t=j+1}^{k}\left(1+I_{t}\right)\right), k=1,2, \cdots,
$$


while (1.3) is equivalent to

$$
U_{k}=u \prod_{j=1}^{k}\left(1+I_{j}\right)+\sum_{j=1}^{k}\left(\left(X_{j}-Y_{j}\right) \prod_{t=j+1}^{k}\left(1+I_{t}\right)\right), k=1,2, \cdots,
$$

where throughout this paper we denote $\prod_{t=a}^{b} x_{t}=1$ and $\sum_{t=a}^{b} x_{t}=0$ if $a>b$.

The effect of interest on ruin probabilities has been discussed by various authors. Sundt and Teugels $(1995,1997)$ have studied the effect of a constant rate of interest on the ruin probability in the compound Poisson risk model. Yang (1998) has considered a special case of (1.5) when $\left\{I_{n}, n=0,1,2, \cdots\right\}$ are identical constants. Cai (2002a) has discussed i.i.d. rates of interest. However, the assumption of constant or i.i.d. rates is not particularly realistic since rates of interest are usually statistically dependent over time. Cai (2002b) has considered a dependent model for rates of interest, in which the rates are assumed to have an $\mathrm{AR}(1)$ structure.

In this paper we consider another dependent model for $\left\{I_{n}, n=0,1,2, \cdots\right\}$, in which $\left\{I_{n}, n=0,1,2, \cdots\right\}$ are assumed to follow a Markov chain. We assume that for all $n=0,1,2, \cdots, I_{n}$ takes a finite or countable number of possible values. This set of possible values is denoted by $\left\{i_{0}, i_{1}, i_{2}, \cdots\right\}$. Suppose that for all $n=0,1,2, \cdots$, and all states $i_{s}, i_{t}, i_{t_{0}}, i_{t_{1}}, \cdots, i_{t_{n-1}}$,

$$
\begin{aligned}
& \operatorname{Pr}\left\{I_{n+1}=i_{t} \mid I_{n}=i_{s}, I_{n-1}=i_{t_{n-1}}, \cdots, I_{1}=i_{t_{1}}, I_{0}=i_{t_{0}}\right\} \\
= & \operatorname{Pr}\left\{I_{n+1}=i_{t} \mid I_{n}=i_{s}\right\}=p_{s t} \geq 0, s, t=0,1,2, \cdots,
\end{aligned}
$$

where $\sum_{t=0}^{\infty} p_{s t}=1$, for $s=0,1,2, \cdots$.

Equation (1.6) means that $\left\{I_{n}, n=0,1,2, \cdots\right\}$ constitute a Markov chain and satisfy the Markov property, i.e. the conditional distribution of any future state $I_{n+1}$ given the past states $I_{0}, I_{1}, I_{2}, \cdots, I_{n-1}$ and the present state $I_{n}$, is independent of the past states and depends only on the present state.

This Markov chain interest model was introduced by Norberg (1997). For a continuoustime Markov chain interest model, see Norberg (1995). Further, $\left\{Y_{n}, n=1,2, \cdots\right\}$, $\left\{X_{n}, n=1,2, \cdots\right\}$, and $\left\{I_{n}, n=0,1,2, \cdots\right\}$ are assumed to be independent.

We define the finite time and ultimate ruin probabilities in model (1.4) with the interest model (1.6), initial surplus $u$, and a given $I_{0}=i_{s}$, respectively, by

$$
\phi_{n}\left(u, i_{s}\right)=\operatorname{Pr}\left\{\bigcup_{k=1}^{n}\left(U_{k}<0\right) \mid I_{0}=i_{s}\right\}
$$




$$
=\operatorname{Pr}\left\{\bigcup_{k=1}^{n}\left(u \prod_{j=1}^{k}\left(1+I_{j}\right)+\sum_{j=1}^{k}\left(X_{j}\left(1+I_{j}\right)-Y_{j}\right) \prod_{t=j+1}^{k}\left(1+I_{t}\right)<0\right) \mid I_{0}=i_{s}\right\}
$$

and

$$
\begin{aligned}
& \phi\left(u, i_{s}\right)=\operatorname{Pr}\left\{\bigcup_{k=1}^{\infty}\left(U_{k}<0\right) \mid I_{0}=i_{s}\right\} \\
= & \operatorname{Pr}\left\{\bigcup_{k=1}^{\infty}\left(u \prod_{j=1}^{k}\left(1+I_{j}\right)+\sum_{j=1}^{k}\left(X_{j}\left(1+I_{j}\right)-Y_{j}\right) \prod_{t=j+1}^{k}\left(1+I_{t}\right)<0\right) \mid I_{0}=i_{s}\right\} .
\end{aligned}
$$

Similarly, we define the finite time and ultimate ruin probabilities in model (1.5) with the interest model (1.6), initial surplus $u$, and a given $I_{0}=i_{s}$, respectively, by

$$
\begin{aligned}
& \varphi_{n}\left(u, i_{s}\right)=\operatorname{Pr}\left\{\bigcup_{k=1}^{n}\left(U_{k}<0\right) \mid I_{0}=i_{s}\right\} \\
= & \operatorname{Pr}\left\{\bigcup_{k=1}^{n}\left(u \prod_{j=1}^{k}\left(1+I_{j}\right)+\sum_{j=1}^{k}\left(X_{j}-Y_{j}\right) \prod_{t=j+1}^{k}\left(1+I_{t}\right)<0\right) \mid I_{0}=i_{s}\right\},
\end{aligned}
$$

and

$$
\begin{aligned}
& \varphi\left(u, i_{s}\right)=\operatorname{Pr}\left\{\bigcup_{k=1}^{\infty}\left(U_{k}<0\right) \mid I_{0}=i_{s}\right\} \\
= & \operatorname{Pr}\left\{\bigcup_{k=1}^{\infty}\left(u \prod_{j=1}^{k}\left(1+I_{j}\right)+\sum_{j=1}^{k}\left(X_{j}-Y_{j}\right) \prod_{t=j+1}^{k}\left(1+I_{t}\right)<0\right) \mid I_{0}=i_{s}\right\} .
\end{aligned}
$$

Thus,

$$
\lim _{n \rightarrow \infty} \phi_{n}\left(u, i_{s}\right)=\phi\left(u, i_{s}\right) \text { and } \lim _{n \rightarrow \infty} \varphi_{n}\left(u, i_{s}\right)=\varphi\left(u, i_{s}\right) .
$$

Like in the cases of constant, i.i.d., and $\operatorname{AR}(1)$ interest rates, it is intuitive (and straightforward to prove) that

$$
\phi\left(u, i_{s}\right) \leq \varphi\left(u, i_{s}\right) \leq \psi(u), u \geq 0
$$

which states that the ruin probability $\psi(u)$ in the classical risk model is reduced by adding interest income to the surplus. Also, (1.8) shows the effects of the timing of payments on the ruin probabilities $\phi\left(u, i_{s}\right)$ and $\varphi\left(u, i_{s}\right)$.

It is very difficult to obtain exact expressions for $\phi\left(u, i_{s}\right)$ and $\varphi\left(u, i_{s}\right)$. An analytic analysis commonly used in ruin theory is to derive inequalities for ruin probabilities. 
For the ruin probability in the classical risk model, we have the well-known Lundberg inequality which states that if $E\left(X_{1}\right)>E\left(Y_{1}\right)$ and there is a unique constant $R>0$ satisfying

$$
E\left[e^{-R\left(X_{1}-Y_{1}\right)}\right]=1
$$

then

$$
\psi(u) \leq e^{-R u}, \quad u \geq 0
$$

Thus, any useful upper bounds for $\phi\left(u, i_{s}\right)$ and $\varphi\left(u, i_{s}\right)$, say

$$
\phi\left(u, i_{s}\right) \leq \Delta\left(u, i_{s}\right) \text { and } \varphi\left(u, i_{s}\right) \leq \Lambda\left(u, i_{s}\right), u \geq 0
$$

should satisfy

$$
\Delta\left(u, i_{s}\right) \leq \Lambda\left(u, i_{s}\right) \leq e^{-R u}, u \geq 0 .
$$

In this paper, we derive probability inequalities for $\phi\left(u, i_{s}\right)$ and $\varphi\left(u, i_{s}\right)$, which both generalize Lundberg's upper bound and satisfy (1.10). In Section 2, we first give recursive equations for $\phi_{n}\left(u, i_{s}\right)$ and $\varphi_{n}\left(u, i_{s}\right)$ and integral equations for $\phi\left(u, i_{s}\right)$ and $\varphi\left(u, i_{s}\right)$. We then derive probability inequalities for $\phi\left(u, i_{s}\right)$ and $\varphi\left(u, i_{s}\right)$ in Section 3 by an inductive approach. In Section 4 , we obtain different probability inequalities for $\phi\left(u, i_{s}\right)$ and $\varphi\left(u, i_{s}\right)$ by a martingale approach. The relationships between Lundberg's inequality and the inequalities derived in this paper are also discussed. Finally, a numerical example is given to illustrate these results in Section 5 .

\section{Recursive and integral equations for ruin probabil- ities}

Throughout this paper, we denote the tail of a distribution function $B$ by $\bar{B}(x)=1-B(x)$. We first give a recursive equation for $\phi_{n}\left(u, i_{s}\right)$ and an integral equation for $\phi\left(u, i_{s}\right)$.

Lemma 2.1 For $n=1,2, \cdots$,

$$
\begin{aligned}
\phi_{n+1}\left(u, i_{s}\right) & =\sum_{t=0}^{\infty} p_{s t} \int_{0}^{\infty} \bar{F}\left((u+x)\left(1+i_{t}\right)\right) d H(x) \\
& +\sum_{t=0}^{\infty} p_{s t} \int_{0}^{\infty} \int_{0}^{(u+x)\left(1+i_{t}\right)} \phi_{n}\left((u+x)\left(1+i_{t}\right)-y, i_{t}\right) d F(y) d H(x)
\end{aligned}
$$


and

$$
\begin{aligned}
\phi\left(u, i_{s}\right) & =\sum_{t=0}^{\infty} p_{s t} \int_{0}^{\infty} \bar{F}\left((u+x)\left(1+i_{t}\right)\right) d H(x) \\
& +\sum_{t=0}^{\infty} p_{s t} \int_{0}^{\infty} \int_{0}^{(u+x)\left(1+i_{t}\right)} \phi\left((u+x)\left(1+i_{t}\right)-y, i_{t}\right) d F(y) d H(x) .
\end{aligned}
$$

Proof. Given $Y_{1}=y, X_{1}=x$, and $I_{1}=i_{t}$, from (1.4), we have

$$
U_{1}=\left(u+X_{1}\right)\left(1+I_{1}\right)-Y_{1}=(u+x)\left(1+i_{t}\right)-y=h-y,
$$

where $h=(u+x)\left(1+i_{t}\right)$. Thus, if $y>h$, then

$$
\operatorname{Pr}\left\{U_{1}<0 \mid Y_{1}=y, X_{1}=x, I_{1}=i_{t}, I_{0}=i_{s}\right\}=1,
$$

which implies that for $y>h$,

$$
\operatorname{Pr}\left\{\bigcup_{k=1}^{n+1}\left(U_{k}<0\right) \mid Y_{1}=y, X_{1}=x, I_{1}=i_{t}, I_{0}=i_{s}\right\}=1,
$$

while if $0 \leq y \leq h$, then

$$
\operatorname{Pr}\left\{U_{1}<0 \mid Y_{1}=y, X_{1}=x, I_{1}=i_{t}, I_{0}=i_{s}\right\}=0 .
$$

Let $\left\{\tilde{Y}_{n}, n=1,2, \cdots\right\},\left\{\tilde{X}_{n}, n=1,2, \cdots\right\}$, and $\left\{\tilde{I}_{n}, n=0,1,2, \cdots\right\}$ be independent copies of $\left\{Y_{n}, n=1,2, \cdots\right\},\left\{X_{n}, n=1,2, \cdots\right\}$, and $\left\{I_{n}, n=0,1,2, \cdots\right\}$, respectively. Thus, (2.1) and (1.4) imply that for $0 \leq y \leq h$,

$$
\begin{aligned}
& \operatorname{Pr}\left\{\bigcup_{k=1}^{n+1}\left(U_{k}<0\right) \mid Y_{1}=y, X_{1}=x, I_{1}=i_{t}, I_{0}=i_{s}\right\} \\
= & \operatorname{Pr}\left\{\bigcup_{k=2}^{n+1}\left(U_{k}<0\right) \mid Y_{1}=y, X_{1}=x, I_{1}=i_{t}, I_{0}=i_{s}\right\} \\
= & \operatorname{Pr}\left\{\bigcup_{k=2}^{n+1}\left((h-y) \prod_{j=2}^{k}\left(1+I_{j}\right)+\sum_{j=2}^{k}\left(X_{j}\left(1+I_{j}\right)-Y_{j}\right) \prod_{t=j+1}^{k}\left(1+I_{t}\right)<0\right) \mid I_{1}=i_{t}\right\} \\
= & \operatorname{Pr}\left\{\bigcup_{k=1}^{n}\left((h-y) \prod_{j=1}^{k}\left(1+\tilde{I}_{j}\right)+\sum_{j=1}^{k}\left(\tilde{X}_{j}\left(1+\tilde{I}_{j}\right)-\tilde{Y}_{j}\right) \prod_{t=j+1}^{k}\left(1+\tilde{I}_{t}\right)<0 \mid \tilde{I}_{0}=i_{t}\right)\right\} \\
= & \phi_{n}\left(h-y, i_{t}\right)=\phi_{n}\left((u+x)\left(1+i_{t}\right)-y, i_{t}\right),
\end{aligned}
$$

where the second equality follows from the Markov property of $\left\{I_{n}, n=0,1,2, \cdots\right\}$ and the independence of $\left\{X_{n}, n=1,2, \cdots\right\},\left\{Y_{n}, n=1,2, \cdots\right\}$, and $\left\{I_{n}, n=0,1,2, \cdots\right\}$. 
Therefore, by conditioning on $Y_{1}, X_{1}$, and $I_{1}$, we get

$$
\begin{aligned}
& \phi_{n+1}\left(u, i_{s}\right)=\operatorname{Pr}\left\{\bigcup_{k=1}^{n+1}\left(U_{k}<0\right) \mid I_{0}=i_{s}\right\} \\
= & \sum_{t=0}^{\infty} p_{s t} \int_{0}^{\infty} \int_{0}^{\infty} \operatorname{Pr}\left\{\bigcup_{k=1}^{n+1}\left(U_{k}<0\right) \mid Y_{1}=y, X_{1}=x, I_{1}=i_{t}, I_{0}=i_{s}\right\} d F(y) d H(x) \\
= & \sum_{t=0}^{\infty} p_{s t} \int_{0}^{\infty} \int_{(u+x)\left(1+i_{t}\right)}^{\infty} d F(y) d H(x) \\
+ & \sum_{t=0}^{\infty} p_{s t} \int_{0}^{\infty} \int_{0}^{(u+x)\left(1+i_{t}\right)} \phi_{n}\left((u+x)\left(1+i_{t}\right)-y, i_{t}\right) d F(y) d H(x) \\
= & \sum_{t=0}^{\infty} p_{s t} \int_{0}^{\infty} \bar{F}\left((u+x)\left(1+i_{t}\right)\right) d H(x) \\
+ & \sum_{t=0}^{\infty} p_{s t} \int_{0}^{\infty} \int_{0}^{(u+x)\left(1+i_{t}\right)} \phi_{n}\left((u+x)\left(1+i_{t}\right)-y, i_{t}\right) d F(y) d H(x)
\end{aligned}
$$

which also yields the integral equation for $\phi\left(u, i_{s}\right)$ in Lemma 2.1 by letting $n \rightarrow \infty$ in (2.2) using the Lebesgue dominated convergence theorem and (1.7).

We now give a recursive equation for $\varphi_{n}\left(u, i_{s}\right)$ and an integral equation for $\varphi\left(u, i_{s}\right)$.

Lemma 2.2 For $n=1,2, \cdots$,

$$
\begin{aligned}
\varphi_{n+1}\left(u, i_{s}\right) & =\sum_{t=0}^{\infty} p_{s t} \int_{0}^{\infty} \bar{F}\left(u\left(1+i_{t}\right)+x\right) d H(x) \\
& +\sum_{t=0}^{\infty} p_{s t} \int_{0}^{\infty} \int_{0}^{u\left(1+i_{t}\right)+x} \varphi_{n}\left(u\left(1+i_{t}\right)+x-y, i_{t}\right) d F(y) d H(x)
\end{aligned}
$$

and

$$
\begin{aligned}
\varphi\left(u, i_{s}\right) & =\sum_{t=0}^{\infty} p_{s t} \int_{0}^{\infty} \bar{F}\left(u\left(1+i_{t}\right)+x\right) d H(x) \\
& +\sum_{t=0}^{\infty} p_{s t} \int_{0}^{\infty} \int_{0}^{u\left(1+i_{t}\right)+x} \varphi\left(u\left(1+i_{t}\right)+x-y, i_{t}\right) d F(y) d H(x)
\end{aligned}
$$

Proof. In this case,

$$
U_{k}=u \prod_{t=1}^{k}\left(1+I_{t}\right)+\sum_{j=1}^{k}\left(X_{j}-Y_{j}\right)\left(\prod_{t=j+1}^{k}\left(1+I_{t}\right)\right) .
$$


By similar arguments to those in the proof of Lemma 2.1 and by conditioning on $Y_{1}, X_{1}$, and $I_{1}$, we get

$$
\begin{aligned}
& \varphi_{n+1}\left(u, i_{s}\right)=\operatorname{Pr}\left\{\bigcup_{k=1}^{n+1}\left(U_{k}<0\right) \mid I_{0}=i_{s}\right\} \\
= & \sum_{t=0}^{\infty} p_{s t} \int_{0}^{\infty} \int_{0}^{\infty} \operatorname{Pr}\left\{\bigcup_{k=1}^{n+1}\left(U_{k}<0\right) \mid Y_{1}=y, X_{1}=x, I_{1}=i_{t}, I_{0}=i_{s}\right\} d F(y) d H(x) \\
= & \sum_{t=0}^{\infty} p_{s t} \int_{0}^{\infty}\left(\int_{u\left(1+i_{t}\right)+x}^{\infty} d F(y)+\int_{0}^{u\left(1+i_{t}\right)+x} \varphi_{n}\left(u\left(1+i_{t}\right)+x-y, i_{t}\right) d F(y)\right) d H(x) \\
= & \sum_{t=0}^{\infty} p_{s t} \int_{0}^{\infty}\left(\bar{F}\left(u\left(1+i_{t}\right)+x\right)+\int_{0}^{u\left(1+i_{t}\right)+x} \varphi_{n}\left(u\left(1+i_{t}\right)+x-y, i_{t}\right) d F(y)\right) d H(x),
\end{aligned}
$$

which also gives the integral equation for $\varphi\left(u, i_{s}\right)$ in Lemma 2.2 by letting $n \rightarrow \infty$.

\section{Probability inequalities by an inductive approach}

Using the recursive equations for $\phi_{n}\left(u, i_{s}\right)$ and $\varphi_{n}\left(u, i_{s}\right)$, we can derive probability inequalities for $\phi\left(u, i_{s}\right)$ and $\varphi\left(u, i_{s}\right)$ by an inductive approach. We first define a generalized adjustment coefficient with the interest model (1.6) and discuss its relationship with the classical adjustment coefficient $R$ given by (1.9).

Proposition 3.1 Let $E\left(X_{1}\right)>E\left(Y_{1}\right)$. Suppose that there exists $R>0$ in (1.9) and $\tau_{s}>0$ satisfying

$$
E\left(e^{\tau_{s} Y_{1}}\right) E\left(e^{-\tau_{s} X_{1}\left(1+I_{1}\right)} \mid I_{0}=i_{s}\right)=1, \quad s=0,1,2, \cdots .
$$

Then,

$$
R_{1}=\min _{0 \leq s<\infty}\left\{\tau_{s}\right\} \geq R
$$

and for all $s=0,1,2, \cdots$,

$$
E\left(e^{R_{1} Y_{1}}\right) E\left(e^{-R_{1} X_{1}\left(1+I_{1}\right)} \mid I_{0}=i_{s}\right) \leq 1 .
$$

Proof. For any $s=0,1,2, \cdots$, by considering the following functions

$$
f_{s}(r)=E\left(e^{r Y_{1}}\right) E\left(e^{-r X_{1}\left(1+I_{1}\right)} \mid I_{0}=i_{s}\right)-1=E\left(e^{r\left(Y_{1}-X_{1}\left(1+I_{1}\right)\right)} \mid I_{0}=i_{s}\right)-1
$$


and

$$
g(r)=E\left(e^{r\left(Y_{1}-X_{1}\right)}\right)-1
$$

we have,

$$
f_{s}^{\prime \prime}(r)=E\left(\left(Y_{1}-X_{1}\left(1+I_{1}\right)\right)^{2} e^{r\left(Y_{1}-X_{1}\left(1+I_{1}\right)\right)} \mid I_{0}=i_{s}\right) \geq 0
$$

which implies that $f_{s}(r)$ is a convex function with $f_{s}(0)=0$ and

$$
f_{s}^{\prime}(0)=E\left(Y_{1}-X_{1}\left(1+I_{1}\right) \mid I_{0}=i_{s}\right) \leq E\left(Y_{1}-X_{1} \mid I_{0}=i_{s}\right)=E\left(Y_{1}-X_{1}\right)<0 .
$$

Similarly, $g(r)$ is a convex function with $g(0)=0$ and $g^{\prime}(0)=E\left(Y_{1}-X_{1}\right)<0$. Therefore, $\tau_{s}$ and $R$ are the unique positive roots of the equations $f_{s}(r)=0$ and $g(r)=0$ respectively on $(0, \infty)$. Furthermore, if $r>0$ and $g(r) \geq 0$, then $r \geq R$; if $0<\tau \leq \tau_{s}$, then $f_{s}(\tau) \leq 0$. However,

$$
1=E\left(e^{\tau_{s}\left(Y_{1}-X_{1}\left(1+I_{1}\right)\right)} \mid I_{0}=i_{s}\right) \leq E\left(e^{\tau_{s}\left(Y_{1}-X_{1}\right)} \mid I_{0}=i_{s}\right)=E\left(e^{\tau_{s}\left(Y_{1}-X_{1}\right)}\right),
$$

i.e. $g\left(\tau_{s}\right) \geq 0$, which implies that $\tau_{s} \geq R$ and $R_{1}=\min _{0 \leq s<\infty} \tau_{s} \geq R$, i.e. (3.2) holds. In addition, for all $s=0,1,2, \cdots, R_{1}=\min _{0 \leq t<\infty} \tau_{t} \leq \tau_{s}$, which implies that $f_{s}\left(R_{1}\right) \leq 0$, i.e. (3.3) holds.

We now obtain a probability inequality for $\phi\left(u, i_{s}\right)$ by an inductive approach.

Theorem 3.1 Under the conditions of Proposition 3.1, for all $s=0,1,2, \cdots$,

$$
\phi\left(u, i_{s}\right) \leq \beta_{1} E\left(e^{R_{1} Y_{1}}\right) E\left(e^{-R_{1}\left(u+X_{1}\right)\left(1+I_{1}\right)} \mid I_{0}=i_{s}\right), \quad u \geq 0
$$

where

$$
\beta_{1}^{-1}=\inf _{t \geq 0} \frac{\int_{t}^{\infty} e^{R_{1} y} d F(y)}{e^{R_{1} t} \bar{F}(t)} .
$$

Proof. For any $x \geq 0$, we have

$$
\begin{aligned}
\vec{F}(x) & =\left(\frac{\int_{x}^{\infty} e^{R_{1} y} d F(y)}{e^{R_{1} x} \bar{F}(x)}\right)^{-1} e^{-R_{1} x} \int_{x}^{\infty} e^{R_{1} y} d F(y) \\
& \leq \beta_{1} e^{-R_{1} x} \int_{x}^{\infty} e^{R_{1} y} d F(y) \\
& \leq \beta_{1} e^{-R_{1} x} E\left(e^{R_{1} Y_{1}}\right)
\end{aligned}
$$


Then, for any $u \geq 0$ and any $i_{s} \geq 0$,

$$
\begin{aligned}
\phi_{1}\left(u, i_{s}\right) & =\operatorname{Pr}\left\{Y_{1}>\left(u+X_{1}\right)\left(1+I_{1}\right) \mid I_{0}=i_{s}\right\} \\
& =\sum_{t=0}^{\infty} p_{s t} \int_{0}^{\infty} \bar{F}\left((u+x)\left(1+i_{t}\right)\right) d H(x)
\end{aligned}
$$

which, together with $x$ replaced by $(u+x)\left(1+i_{t}\right)$ in $(3.8)$, implies that

$$
\begin{aligned}
\phi_{1}\left(u, i_{s}\right) & \leq \sum_{t=0}^{\infty} p_{s t}\left(\beta_{1} E\left(e^{R_{1} Y_{1}}\right) \int_{0}^{\infty} e^{-R_{1}(u+x)\left(1+i_{t}\right)} d H(x)\right) \\
& \leq \beta_{1} E\left(e^{R_{1} Y_{1}}\right) \sum_{t=0}^{\infty} p_{s t} \int_{0}^{\infty} e^{-R_{1}(u+x)\left(1+i_{t}\right)} d H(x) \\
& =\beta_{1} E\left(e^{R_{1} Y_{1}}\right) E\left(e^{-R_{1}\left(u+X_{1}\right)\left(1+I_{1}\right)} \mid I_{0}=i_{s}\right) .
\end{aligned}
$$

Under an inductive hypothesis, we assume for any $u \geq 0$ and any $i_{s} \geq 0$,

$$
\phi_{n}\left(u, i_{s}\right) \leq \beta_{1} E\left(e^{R_{1} Y_{1}}\right) E\left(e^{-R_{1}\left(u+X_{1}\right)\left(1+I_{1}\right)} \mid I_{0}=i_{s}\right) .
$$

Thus, for $0 \leq y \leq(u+x)\left(1+i_{t}\right)$, with $u$ and $i_{s}$ replaced by $(u+x)\left(1+i_{t}\right)-y$ and $i_{t}$ respectively in (3.9), $I_{1} \geq 0$, and (3.3), we have

$$
\begin{aligned}
& \phi_{n}\left((u+x)\left(1+i_{t}\right)-y, i_{t}\right) \\
\leq & \beta_{1} E\left(e^{R_{1} Y_{1}}\right) E\left(e^{-R_{1}\left((u+x)\left(1+i_{t}\right)-y+X_{1}\right)\left(1+I_{1}\right)} \mid I_{0}=i_{t}\right) \\
= & \beta_{1} E\left(e^{R_{1} Y_{1}}\right) E\left(e^{-R_{1}\left((u+x)\left(1+i_{t}\right)-y\right)\left(1+I_{1}\right)-R_{1} X_{1}\left(1+I_{1}\right)} \mid I_{0}=i_{t}\right) \\
\leq & \beta_{1} E\left(e^{R_{1} Y_{1}}\right) E\left(e^{-R_{1} X_{1}\left(1+I_{1}\right)} \mid I_{0}=i_{t}\right) e^{-R_{1}\left((u+x)\left(1+i_{t}\right)-y\right)} \\
\leq & \beta_{1} e^{-R_{1}\left((u+x)\left(1+i_{t}\right)-y\right)} .
\end{aligned}
$$

Therefore, by Lemma 2.1, (3.7), and (3.10), we get

$$
\begin{aligned}
\phi_{n+1}\left(u, i_{s}\right) & \leq \sum_{t=0}^{\infty} p_{s t}\left(\beta_{1} \int_{0}^{\infty} e^{-R_{1}(u+x)\left(1+i_{t}\right)} \int_{(u+x)\left(1+i_{t}\right)}^{\infty} e^{R_{1} y} d F(y) d H(x)\right) \\
& +\sum_{t=0}^{\infty} p_{s t}\left(\beta_{1} \int_{0}^{\infty} e^{-R_{1}(u+x)\left(1+i_{t}\right)} \int_{0}^{(u+x)\left(1+i_{t}\right)} e^{R_{1} y} d F(y) d H(x)\right) \\
& =\sum_{t=0}^{\infty} p_{s t}\left(\beta_{1} \int_{0}^{\infty} e^{-R_{1}(u+x)\left(1+i_{t}\right)} \int_{0}^{\infty} e^{R_{1} y} d F(y) d H(x)\right) \\
& =\beta_{1} E\left(e^{R_{1} Y_{1}}\right) \sum_{t=0}^{\infty} p_{s t} \int_{0}^{\infty} e^{-R_{1}(u+x)\left(1+i_{t}\right)} d H(x) \\
& =\beta_{1} E\left(e^{R_{1} Y_{1}}\right) E\left(e^{-R_{1}\left(u+X_{1}\right)\left(1+I_{1}\right)} \mid I_{0}=i_{s}\right) .
\end{aligned}
$$


Hence, for any $n=1,2, \cdots,(3.9)$ holds. Therefore, (3.5) follows by letting $n \rightarrow \infty$ in (3.9).

Similarly, we can obtain the following probability inequality for $\varphi\left(u, i_{s}\right)$.

Theorem 3.2 Let $R>0$ be the constant satisfying (1.9). Then,

$$
\varphi\left(u, i_{s}\right) \leq \beta E\left(e^{-R u\left(1+I_{1}\right)} \mid I_{0}=i_{s}\right), u \geq 0
$$

where

$$
\beta^{-1}=\inf _{t \geq 0} \frac{\int_{t}^{\infty} e^{R y} d F(y)}{e^{R t} \bar{F}(t)}
$$

Proof. Similarly to (3.7) and (3.8), we have for any $x \geq 0$,

$$
\begin{aligned}
\bar{F}(x) & \leq \beta e^{-R x} \int_{x}^{\infty} e^{R y} d F(y) \\
& \leq \beta e^{-R x} E\left(e^{R Y_{1}}\right) .
\end{aligned}
$$

Then, for any $u \geq 0$ and any $i_{s} \geq 0$,

$$
\begin{aligned}
\varphi_{1}\left(u, i_{s}\right) & =\operatorname{Pr}\left\{Y_{1}>u\left(1+I_{1}\right)+X_{1} \mid I_{0}=i_{s}\right\} \\
& =\sum_{t=0}^{\infty} p_{s t} \int_{0}^{\infty} \bar{F}\left(u\left(1+i_{t}\right)+x\right) d H(x)
\end{aligned}
$$

which implies by (3.14) that

$$
\begin{aligned}
\varphi_{1}\left(u, i_{s}\right) & \leq \sum_{t=0}^{\infty} p_{s t}\left(\beta E\left(e^{R Y_{1}}\right) \int_{0}^{\infty} e^{-R\left(u\left(1+i_{t}\right)+x\right)} d H(x)\right) \\
& =\beta E\left(e^{R Y_{1}}\right) \sum_{t=0}^{\infty} p_{s t} \int_{0}^{\infty} e^{-R\left(u\left(1+i_{t}\right)+x\right)} d H(x) \\
& =\beta E\left(e^{R Y_{1}}\right) E\left(e^{-R\left(u\left(1+I_{1}\right)+X_{1}\right)} \mid I_{0}=i_{s}\right) \\
& =\beta E\left(e^{R Y_{1}}\right) E\left(e^{-R X_{1}}\right) E\left(e^{-R u\left(1+I_{1}\right)} \mid I_{0}=i_{s}\right) \\
& =\beta E\left(e^{-R u\left(1+I_{1}\right)} \mid I_{0}=i_{s}\right) .
\end{aligned}
$$

Under an inductive hypothesis, we assume for any $u \geq 0$ and any $i_{s} \geq 0$,

$$
\varphi_{n}\left(u, i_{s}\right) \leq \beta E\left(e^{-R u\left(1+I_{1}\right)} \mid I_{0}=i_{s}\right) .
$$


Thus, for $0 \leq y \leq u\left(1+i_{t}\right)+x$, with $u$ and $i_{s}$ replaced by $u\left(1+i_{t}\right)+x-y$ and $i_{t}$ respectively in (3.15), and $I_{1} \geq 0$, we have

$$
\begin{aligned}
\varphi_{n}\left(u\left(1+i_{t}\right)+x-y, i_{t}\right) & \leq \beta E\left(e^{-R\left(u\left(1+i_{t}\right)+x-y\right)\left(1+I_{1}\right)} \mid I_{0}=i_{t}\right) \\
& \leq \beta e^{-R\left(u\left(1+i_{t}\right)+x-y\right)} .
\end{aligned}
$$

Therefore, by Lemma 2.2, (3.13), and (3.16), we get

$$
\begin{aligned}
\varphi_{n+1}\left(u, i_{s}\right) & \leq \sum_{t=0}^{\infty} p_{s t}\left(\beta \int_{0}^{\infty} e^{-R\left(u\left(1+i_{t}\right)+x\right)} \int_{u\left(1+i_{t}\right)+x}^{\infty} e^{R y} d F(y) d H(x)\right) \\
& +\sum_{t=0}^{\infty} p_{s t}\left(\beta \int_{0}^{\infty} \int_{0}^{u\left(1+i_{t}\right)+x} e^{-R\left(u\left(1+i_{t}\right)+x-y\right)} d F(y) d H(x)\right) \\
& =\sum_{t=0}^{\infty} p_{s t}\left(\beta \int_{0}^{\infty} e^{-R\left(u\left(1+i_{t}\right)+x\right)} \int_{0}^{\infty} e^{R y} d F(y) d H(x)\right) \\
& =\beta E\left(e^{R Y_{1}}\right) \sum_{t=0}^{\infty} p_{s t} \int_{0}^{\infty} e^{-R\left(u\left(1+i_{t}\right)+x\right)} d H(x) \\
& =\beta E\left(e^{R Y_{1}}\right) E\left(e^{-R X_{1}}\right) E\left(e^{-R u\left(1+I_{1}\right)} \mid I_{0}=i_{s}\right) \\
& =\beta E\left(e^{-R u\left(1+I_{1}\right)} \mid I_{0}=i_{s}\right) .
\end{aligned}
$$

Hence, for any $n=1,2, \cdots,(3.15)$ holds. Therefore, (3.11) follows by letting $n \rightarrow \infty$ in (3.15).

Refinements of the upper bounds in Theorems 3.1 and 3.2 can be obtained when $F$ is new worse than used in convex ordering (NWUC). A lifetime distribution $B$ is said to be NWUC if for all $x \geq 0, y \geq 0$,

$$
\int_{x+y}^{\infty} \bar{B}(t) d t \geq \bar{B}(x) \int_{y}^{\infty} \bar{B}(t) d t
$$

The class of NWUC distributions is larger than the class of decreasing failure rate distributions. See Shaked and Shanthikumar (1994) for properties of NWUC and other classes of lifetime distributions.

Corollary 3.1 Under the conditions of Theorems 3.1 and 3.2, if $F$ is NWUC, then,

$$
\phi\left(u, i_{s}\right) \leq E\left(e^{-R_{1}\left(u+X_{1}\right)\left(1+I_{1}\right)} \mid I_{0}=i_{s}\right), u \geq 0
$$

and

$$
\varphi\left(u, i_{s}\right) \leq\left(E\left(e^{R Y_{1}}\right)\right)^{-1} E\left(e^{-R u\left(1+I_{1}\right)} \mid I_{0}=i_{s}\right), u \geq 0
$$


Proof. By Proposition 6.1.1 of Willmot and Lin (2001), we know that if $F$ is NWUC, then $\beta_{1}^{-1}=E\left(e^{R_{1} Y_{1}}\right)$ and $\beta^{-1}=E\left(e^{R Y_{1}}\right)$. Thus (3.17) and (3.18) follow from (3.5) and (3.11), respectively.

We denote the upper bound in Theorem 3.1 by $A\left(u, i_{s}\right)$, i.e.

$$
A\left(u, i_{s}\right)=\beta_{1} E\left(e^{R_{1} Y_{1}}\right) E\left(e^{-R_{1}\left(u+X_{1}\right)\left(1+I_{1}\right)} \mid I_{0}=i_{s}\right),
$$

and denote the upper bound in Theorem 3.2 by $B\left(u, i_{s}\right)$, i.e.

$$
B\left(u, i_{s}\right)=\beta E\left(e^{-R u\left(1+I_{1}\right)} \mid I_{0}=i_{s}\right) .
$$

Proposition 3.2 For any $u \geq 0$,

$$
A\left(u, i_{s}\right) \leq e^{-R u} \text { and } B\left(u, i_{s}\right) \leq e^{-R u} .
$$

Further, if $F$ is NWUC, then for any $u \geq 0$,

$$
A\left(u, i_{s}\right) \leq B\left(u, i_{s}\right) \leq e^{-R u}
$$

Proof. First, by $I_{1} \geq 0,(3.2),(3.3)$, and $0 \leq \beta_{1} \leq 1$, we have for $u \geq 0$,

$$
\begin{aligned}
A\left(u, i_{s}\right) & =\beta_{1} E\left(e^{R_{1} Y_{1}}\right) E\left(e^{-R_{1} u\left(1+I_{1}\right)-R_{1} X_{1}\left(1+I_{1}\right)} \mid I_{0}=i_{s}\right) \\
& \leq \beta_{1} E\left(e^{R_{1} Y_{1}}\right) E\left(e^{-R_{1} u-R_{1} X_{1}\left(1+I_{1}\right)} \mid I_{0}=i_{s}\right) \\
& =\beta_{1} e^{-R_{1} u} E\left(e^{R_{1} Y_{1}}\right) E\left(e^{-R_{1} X_{1}\left(1+I_{1}\right)} \mid I_{0}=i_{s}\right) \\
& \leq e^{-R u}
\end{aligned}
$$

Then, $0 \leq \beta \leq 1$ implies that for $u \geq 0$,

$$
B\left(u, i_{s}\right)=\beta e^{-R u} E\left(e^{-R I_{1} u} \mid I_{0}=i_{s}\right) \leq e^{-R u} .
$$

Further, by (3.2) we have that for any $t \geq 0$,

$$
\frac{\int_{t}^{\infty} e^{R_{1} y} d F(y)}{e^{R_{1} t} \bar{F}(t)}=\frac{\int_{t}^{\infty} e^{R_{1}(y-t)} d F(y)}{\bar{F}(t)} \geq \frac{\int_{t}^{\infty} e^{R(y-t)} d F(y)}{\bar{F}(t)}
$$

which, using (3.6) and (3.12), implies that

$$
\beta_{1}^{-1} \geq \beta^{-1}, \text { or } \beta_{1} \leq \beta .
$$


Thus, if $F$ is NWUC, by Corollary 3.1, (3.2), and (1.9), we have for $u \geq 0$,

$$
\begin{aligned}
A\left(u, i_{s}\right) & =E\left(e^{-R_{1} X_{1}\left(1+I_{1}\right)} e^{-R_{1} u\left(1+I_{1}\right)} \mid I_{0}=i_{s}\right) \\
& \leq E\left(e^{-R X_{1}\left(1+I_{1}\right)} e^{-R u\left(1+I_{1}\right)} \mid I_{0}=i_{s}\right) \\
& \leq E\left(e^{-R X_{1}}\right) E\left(e^{-R u\left(1+I_{1}\right)} \mid I_{0}=i_{s}\right) \\
& =\left(E\left(e^{R Y_{1}}\right)\right)^{-1} E\left(e^{-R u\left(1+I_{1}\right)} \mid I_{0}=i_{s}\right) \\
& =B\left(u, i_{s}\right) .
\end{aligned}
$$

Hence, (3.19) holds.

Proposition 3.2 means that the upper bounds in Theorem 3.1 and 3.2 are less than the Lundberg upper bound while the upper bound in Theorem 3.1 for $\phi\left(u, i_{s}\right)$ is less than the upper bound in Theorem 3.2 for $\varphi\left(u, i_{s}\right)$ if $F$ is NWUC.

We remark that although our results apply when $I_{n}$ takes a countable number of values, in practice we would apply a model under which $I_{n}$ takes a finite number of values. This practice allows calculation of the constants $\left\{\tau_{s}\right\}$ and hence $R_{1}$. The same comment applies to the constants $R_{2}$ and $R_{3}$ which are defined in the next section.

Further, if $I_{s}=0$ for all $s=0,1,2, \cdots$, then $R_{1}=\tau_{s}=R$ and $\beta_{1}=\beta$. Thus, the upper bounds in Theorems 3.1 and 3.2 reduce to $\beta e^{-R u}$, which yields an improvement on the Lundberg upper bound since $0 \leq \beta \leq 1$. For further refinements of the Lundberg inequality in different applied probability models, see Grandell (1991), Willmot et al (2001), Willmot and Lin (2001), and references therein.

\section{Probability inequalities by the martingale approach}

Another tool for deriving probability inequalities for ruin probabilities is the martingale approach. The ruin probability associated with either the risk process given by (1.4) or by (1.5) is equal to the ruin probability associated with its discounted risk process $\left\{V_{n}, n=1,2, \cdots\right\}$, i.e.

$$
\operatorname{Pr}\left\{\bigcup_{k=1}^{n}\left(U_{k}<0\right) \mid I_{0}=i_{s}\right\}=\operatorname{Pr}\left\{\bigcup_{k=1}^{n}\left(V_{k}<0\right) \mid I_{0}=i_{s}\right\},
$$


where

$$
V_{k}=U_{k} \prod_{j=1}^{k}\left(1+I_{j}\right)^{-1}, \quad k=1,2, \cdots .
$$

In the classical risk model, $\left\{e^{-R U_{n}}, n=1,2, \cdots\right\}$ is a martingale. However, for the generalized risk processes (1.4) and (1.5), there is no constant $r>0$ such that $\left\{e^{-r U_{n}}, n=1,2, \cdots\right\}$ is a martingale. However, there exists a constant $r>0$ such that $\left\{e^{-r V_{n}}, n=1,2, \cdots\right\}$ is a supermartingale, which allows us to derive probability inequalities by the optional stopping theorem. Such a constant is defined in the following proposition.

Proposition 4.1 Let $E\left(X_{1}\right)>E\left(Y_{1}\right)$. Suppose that there exists $R>0$ satisfying (1.9) and there exists $\kappa_{s}>0$ satisfying

$$
E\left(e^{-\kappa_{s}\left(X_{1}-Y_{1}\left(1+I_{1}\right)^{-1}\right)} \mid I_{0}=i_{s}\right)=1, \quad s=0,1,2, \cdots .
$$

Then,

$$
R_{2}=\min _{0 \leq s<\infty}\left\{\kappa_{s}\right\} \geq R
$$

and for all $s=0,1,2, \cdots$,

$$
E\left(e^{-R_{2}\left(X_{1}-Y_{1}\left(1+I_{1}\right)^{-1}\right)} \mid I_{0}=i_{s}\right) \leq 1 .
$$

Proof. Similarly to Proposition 3.1, for any $s=0,1,2, \cdots$, we know that

$$
h_{s}(r)=E\left(e^{r\left(Y_{1}\left(1+I_{1}\right)^{-1}-X_{1}\right)} \mid I_{0}=i_{s}\right)-1
$$

is a convex function with $h_{s}(0)=0$ and

$$
h_{s}^{\prime}(0)=E\left(Y_{1}\left(1+I_{1}\right)^{-1}-X_{1} \mid I_{0}=i_{s}\right) \leq E\left(Y_{1}-X_{1} \mid I_{0}=i_{s}\right)=E\left(Y_{1}-X_{1}\right)<0 .
$$

Therefore, $\kappa_{s}$ is the unique positive root of the equation $h_{s}(r)=0$ on $(0, \infty)$. Further, if $0<\kappa \leq \kappa_{s}$, then $h_{s}(\kappa) \leq 0$. However,

$$
1=E\left(e^{\kappa_{s}\left(Y_{1}\left(1+I_{1}\right)^{-1}-X_{1}\right)} \mid I_{0}=i_{s}\right) \leq E\left(e^{\kappa_{s}\left(Y_{1}-X_{1}\right)} \mid I_{0}=i_{s}\right)=E\left(e^{\kappa_{s}\left(Y_{1}-X_{1}\right)}\right)
$$

or $g\left(\kappa_{s}\right) \geq 0$, where $g(r)$ is defined in (3.4). Hence, $\kappa_{s} \geq R$ and $R_{2}=\min _{0 \leq s<\infty} \kappa_{s} \geq R$, i.e. (4.2) holds. In addition, for all $s=0,1,2, \cdots, R_{2}=\min _{0 \leq t<\infty} \kappa_{t} \leq \kappa_{s}$, which implies that $h_{s}\left(R_{2}\right) \leq 0$, i.e. (4.3) holds. 
Theorem 4.1 Under the conditions of Proposition 4.1, for all $s=0,1,2, \cdots$,

$$
\phi\left(u, i_{s}\right) \leq e^{-R_{2} u}, \quad u \geq 0
$$

Proof. For the process $\left\{U_{k}\right\}$ given by (1.4), we denote

$$
V_{k}=U_{k} \prod_{j=1}^{k}\left(1+I_{j}\right)^{-1}=u+\sum_{j=1}^{k}\left(\left(X_{j}\left(1+I_{j}\right)-Y_{j}\right) \prod_{t=1}^{j}\left(1+I_{t}\right)^{-1}\right)
$$

and $S_{n}=e^{-R_{2} V_{n}}$. Then

$$
S_{n+1}=S_{n} e^{-R_{2}\left(X_{n+1}-Y_{n+1}\left(1+I_{n+1}\right)^{-1}\right)} \prod_{t=1}^{n}\left(1+I_{t}\right)^{-1} .
$$

Thus, for any $n \geq 1$,

$$
\begin{aligned}
& E\left(S_{n+1} \mid X_{1}, \cdots, X_{n}, Y_{1}, \cdots, Y_{n}, I_{1}, \cdots, I_{n}\right) \\
& =S_{n} E\left(e^{-R_{2}\left(X_{n+1}-Y_{n+1}\left(1+I_{n+1}\right)^{-1}\right)} \prod_{t=1}^{n}\left(1+I_{t}\right)^{-1} \mid X_{1}, \cdots, X_{n}, Y_{1}, \cdots, Y_{n}, I_{1}, \cdots, I_{n}\right) \\
& =S_{n} E\left(e^{-R_{2}\left(X_{n+1}-Y_{n+1}\left(1+I_{n+1}\right)^{-1}\right)} \prod_{t=1}^{n}\left(1+I_{t}\right)^{-1} \mid I_{1}, \cdots, I_{n}\right) \\
& \leq S_{n}\left(E\left(e^{-R_{2}\left(X_{n+1}-Y_{n+1}\left(1+I_{n+1}\right)^{-1}\right)} \mid I_{1}, \cdots, I_{n}\right) \prod_{t=1}^{n}\left(1+I_{t}\right)^{-1}\right. \\
& =S_{n} \text {, }
\end{aligned}
$$

where the inequality follows from $0 \leq \prod_{t=1}^{n}\left(1+I_{t}\right)^{-1} \leq 1$ and Jensen's inequality while the last equality follows from

$$
\begin{aligned}
& E\left(e^{-R_{2}\left(X_{n+1}-Y_{n+1}\left(1+I_{n+1}\right)^{-1}\right)} \mid I_{1}, \cdots, I_{n}\right)=E\left(e^{-R_{2}\left(X_{n+1}-Y_{n+1}\left(1+I_{n+1}\right)^{-1}\right)} \mid I_{n}\right) \\
= & E\left(e^{-R_{2}\left(X_{1}-Y_{1}\left(1+I_{1}\right)^{-1}\right)} \mid I_{0}\right) \leq 1 .
\end{aligned}
$$

Hence, $\left\{S_{n}, \quad n=1,2, \cdots\right\}$ is a supermartingale with respect to the filtration $\mathcal{F}_{n}=\sigma\left\{X_{1}, \cdots, X_{n}, Y_{1}, \cdots, Y_{n}, I_{1}, \cdots, I_{n}\right\}$.

Let $T_{s}=\min \left\{n: V_{n}<0 \mid I_{0}=i_{s}\right\}$, where $V_{n}$ is given by (4.6). Then $T_{s}$ is a stopping time and $n \wedge T_{s}=\min \left(n, T_{s}\right)$ is a finite stopping time. Thus, by the optional stopping theorem for supermartingales, we get

$$
E\left(S_{n \wedge T_{s}}\right) \leq E\left(S_{0}\right)=e^{-R_{2} u}
$$


Hence,

$$
\begin{aligned}
e^{-R_{2} u} & \geq E\left(S_{n \wedge T_{s}}\right) \geq E\left(S_{n \wedge T_{s}} I\left(T_{s} \leq n\right)\right) \\
& =E\left(S_{T_{s}} I\left(T_{s} \leq n\right)\right) \\
& =E\left(e^{\left.-R_{2} V_{T_{s}} I\left(T_{s} \leq n\right)\right)}\right. \\
& \geq E\left(I\left(T_{s} \leq n\right)\right) \\
& =\operatorname{Pr}\left\{T_{s} \leq n\right\}=\phi_{n}\left(u, i_{s}\right)
\end{aligned}
$$

where (4.7) follows from $V_{T_{s}}<0$ and (4.8) follows from

$$
\phi_{n}\left(u, i_{s}\right)=\operatorname{Pr}\left\{\bigcup_{k=1}^{n}\left(U_{k}<0\right) \mid I_{0}=i_{s}\right\}=\operatorname{Pr}\left\{\bigcup_{k=1}^{n}\left(V_{k}<0\right) \mid I_{0}=i_{s}\right\}=\operatorname{Pr}\left\{T_{s} \leq n\right\} .
$$

Thus, (4.5) follows by letting $n \rightarrow \infty$ in (4.8).

Proposition 4.2 Let $E\left(X_{1}\right)>E\left(Y_{1}\right)$. Suppose that there exists $R>0$ satisfying (1.9) and there exists $\rho_{s}>0$ satisfying

$$
E\left(e^{-\rho_{s}\left(X_{1}-Y_{1}\right)\left(1+I_{1}\right)^{-1}} \mid I_{0}=i_{s}\right)=1, \quad s=0,1,2, \cdots .
$$

Then,

$$
R \leq R_{3}=\min _{0 \leq s<\infty}\left\{\rho_{s}\right\} \leq R_{2}
$$

and for all $s=0,1,2, \cdots$,

$$
E\left(e^{\left.-R_{3}\left(X_{1}-Y_{1}\right)\left(1+I_{1}\right)^{-1}\right)} \mid I_{0}=i_{s}\right) \leq 1 .
$$

Proof. For any $s=0,1,2, \cdots$, we know that

$$
l_{s}(r)=E\left(e^{r\left(Y_{1}-X_{1}\right)\left(1+I_{1}\right)^{-1}} \mid I_{0}=i_{s}\right)-1
$$

is a convex function with $l_{s}(0)=0$ and

$$
\begin{aligned}
l_{s}^{\prime}(0) & =E\left(\left(Y_{1}-X_{1}\right)\left(1+I_{1}\right)^{-1} \mid I_{0}=i_{s}\right) \\
& =E\left(Y_{1}-X_{1} \mid I_{0}=i_{s}\right) E\left(\left(1+I_{1}\right)^{-1} \mid I_{0}=i_{s}\right) \\
& =E\left(Y_{1}-X_{1}\right) E\left(\left(1+I_{1}\right)^{-1} \mid I_{0}=i_{s}\right)<0 .
\end{aligned}
$$


Therefore, $\rho_{s}$ is the unique positive root of the equation $l_{s}(r)=0$ on $(0, \infty)$. Further, if $0<\rho \leq \rho_{s}$, then $l_{s}(\rho) \leq 0$. However,

$$
1=E\left(e^{\rho_{s}\left(Y_{1}-X_{1}\right)\left(1+I_{1}\right)^{-1}} \mid I_{0}=i_{s}\right) \geq E\left(e^{\rho_{s}\left(Y_{1}\left(1+I_{1}\right)^{-1}-X_{1}\right)} \mid I_{0}=i_{s}\right),
$$

or $h_{s}\left(\rho_{s}\right) \leq 0$, which implies that $\rho_{s} \leq \kappa_{s}$, where $h_{s}(r)$ and $\kappa_{s}$ are given by (4.4) and (4.1), respectively. Therefore,

$$
R_{3}=\min _{0 \leq s<\infty} \rho_{s} \leq \min _{0 \leq s<\infty} \kappa_{s}=R_{2}
$$

Further, by Jensen's inequality and (1.9), we have

$$
\begin{aligned}
E\left(e^{R\left(Y_{1}-X_{1}\right)\left(1+I_{1}\right)^{-1}} \mid I_{0}=i_{s}\right) & =\sum_{t=0}^{\infty} p_{s t} E\left(e^{R\left(Y_{1}-X_{1}\right)\left(1+i_{t}\right)^{-1}}\right) \\
\leq \sum_{t=0}^{\infty} p_{s t}\left(E\left(e^{R\left(Y_{1}-X_{1}\right)}\right)\right)^{\left(1+i_{t}\right)^{-1}} & =\sum_{t=0}^{\infty} p_{s t}=1,
\end{aligned}
$$

which implies that $l_{s}(R) \leq 0$. Hence $R \leq \rho_{s}$ and $R_{3}=\min _{0 \leq s<\infty} \rho_{s} \geq R$. Thus, (4.10) holds. In addition, for all $s=0,1,2, \cdots, R_{3}=\min _{0 \leq t<\infty} \rho_{t} \leq \rho_{s}$, which implies that $l_{s}\left(R_{3}\right) \leq 0$, i.e. (4.11) holds.

Theorem 4.2 Under the conditions of Proposition 4.2, for all $s=0,1,2, \cdots$,

$$
\varphi\left(u, i_{s}\right) \leq e^{-R_{3} u}, \quad u \geq 0 .
$$

Proof. For the process $\left\{U_{k}\right\}$ given by (1.5), we denote

$$
V_{k}=U_{k} \prod_{j=1}^{k}\left(1+I_{j}\right)^{-1}=u+\sum_{j=1}^{k}\left(\left(X_{j}-Y_{j}\right) \prod_{t=1}^{j}\left(1+I_{t}\right)^{-1}\right)
$$

and $S_{n}=e^{-R_{3} V_{n}}$. Then

$$
S_{n+1}=S_{n} e^{-R_{3}\left(X_{n+1}-Y_{n+1}\right) \prod_{t=1}^{n+1}\left(1+I_{t}\right)^{-1}} .
$$

Thus, for any $n \geq 1$,

$$
\begin{aligned}
& E\left(S_{n+1} \mid X_{1}, \cdots, X_{n}, Y_{1}, \cdots, Y_{n}, I_{1}, \cdots, I_{n}\right) \\
= & S_{n} E\left(e^{-R_{3}\left(X_{n+1}-Y_{n+1}\right) \prod_{t=1}^{n+1}\left(1+I_{t}\right)^{-1}} \mid X_{1}, \cdots, X_{n}, Y_{1}, \cdots, Y_{n}, I_{1}, \cdots, I_{n}\right) \\
= & S_{n} E\left(e^{-R_{3}\left(X_{n+1}-Y_{n+1}\right)\left(1+I_{n+1}\right)^{-1} \prod_{t=1}^{n}\left(1+I_{t}\right)^{-1}} \mid I_{1}, \cdots, I_{n}\right) \\
\leq & S_{n}\left(E\left(e^{-R_{3}\left(X_{n+1}-Y_{n+1}\right)\left(1+I_{n+1}\right)^{-1}} \mid I_{1}, \cdots, I_{n}\right)\right) \prod_{t=1}^{n}\left(1+I_{t}\right)^{-1} \\
\leq & S_{n}
\end{aligned}
$$


which implies that $\left\{S_{n}, n=1,2, \cdots\right\}$ is a supermartingale. Let $T_{s}=\min \left\{n: V_{n}<0 \mid I_{0}=\right.$ $i_{s}$ \} where $V_{n}$ is given by (4.13). Then $T_{s}$ is a stopping time and $n \wedge T_{s}=\min \left(n, T_{s}\right)$ is a finite stopping time. Thus, (4.12) follows from the same arguments as those for (4.5).

It is clear that the upper bounds derived by the martingale approach satisfy (1.10), i.e.

$$
e^{-R_{2} u} \leq e^{-R_{3} u} \leq e^{-R u}, u \geq 0
$$

since (4.10) holds. In addition, if for all $s=0,1,2, \cdots, I_{s}=0$, then $R_{2}=R_{3}=R$ and the upper bounds in Theorems 4.1 and 4.2 reduce to Lundberg's upper bound.

It seems that the inequalities in Section 3 cannot be derived by the martingale approach. In addition, like the case of i.i.d. rates of interest discussed by Cai (2002a), the numerical results of Section 5 suggest that upper bounds derived by the inductive approach of Section 3 are tighter than those obtained by the martingale approach of this section.

\section{Numerical illustration}

In this section we give a numerical example to illustrate the bounds derived in Sections 3 and 4 . Without loss of generality we can work in monetary units equal to $E\left(Y_{1}\right)$. Let $Y_{1}$ have a gamma distribution with each parameter equal to $1 / 2$, so that $E\left(Y_{1}\right)=1$ and $V\left(Y_{1}\right)=2$. Let $\operatorname{Pr}\left(X_{1}=1.1\right)=1$ so that there is deterministic premium income with a loading of $10 \%$. Consider an interest model with three possible interest rates: $i_{0}=6 \%$, $i_{1}=8 \%$ and $i_{2}=10 \%$. Let $\mathbf{P}=\left\{p_{s t}\right\}$ be given by

$$
\mathbf{P}=\left(\begin{array}{ccc}
0.2 & 0.8 & 0 \\
0.15 & 0.7 & 0.15 \\
0 & 0.8 & 0.2
\end{array}\right)
$$

Thus, our interest rate model incorporates mean reversion to a level of $8 \%$.

We can easily find that the constant $R$ which satisfies (1.9) is $R=0.08807$. Similarly, we find that $R_{1}=0.14665$ by solving equation (3.1) for $\tau_{0}, \tau_{1}$ and $\tau_{2}$, where, for example, the equation satisfied by $\tau_{1}$ is

$$
0.15 e^{-1.166 \tau_{1}}+0.7 e^{-1.188 \tau_{1}}+0.15 e^{-1.21 \tau_{1}}=\left(1-2 \tau_{1}\right)^{1 / 2} .
$$


As our gamma distribution is DFR and hence NWUC, we can apply the results of Corollary 3.1 with, for example,

$$
\phi\left(u, i_{1}\right) \leq 0.15 e^{-1.06 R_{1}(u+1.1)}+0.7 e^{-1.08 R_{1}(u+1.1)}+0.15 e^{-1.1 R_{1}(u+1.1)} .
$$

Table 1 shows values of $\phi\left(u, i_{1}\right), \varphi\left(u, i_{1}\right)$ and $e^{-R u}$ for a range of values of $u$. We note that the upper bounds are ordered in accordance with Proposition 3.2.

Similarly, we can find from equations (4.1) and (4.9) that $R_{2}=0.15773$ and $R_{3}=$ 0.09475 , where, for example, we find $\kappa_{1}$ as the solution of

$$
e^{-1.1 \kappa_{1}}\left(0.15 M_{Y}\left(\kappa_{1} / 1.06\right)+0.7 M_{Y}\left(\kappa_{1} / 1.08\right)+0.15 M_{Y}\left(\kappa_{1} / 1.1\right)\right)=1
$$

where $M_{Y}(t)=(1-2 t)^{-1 / 2}$. Table 2 shows values of $e^{-R_{2} u}, e^{-R_{3} u}$ and $e^{-R u}$ for a range of values of $u$. The ordering of these bounds is as expected, and we note that $\phi\left(u, i_{1}\right)<e^{-R_{2} u}$ and $\varphi\left(u, i_{1}\right)<e^{-R_{3} u}$ in line with our comments in Section 4.

\section{References}

[1] Asmussen, S. (2000) Ruin Probabilities. World Scientific, Singapore.

[2] Cai, J. (2002a) Discrete time risk models under rates of interest. Probability in the Engineering and Informational Sciences, 16, 309-324.

[3] Cai, J. (2002b) Ruin probabilities with dependent rates of interest. Journal of Applied Probability, 39, 312-323.

[4] Grandell, J. (1991) Aspects of Risk Theory. Springer-Verlag, New York.

[5] Norberg, R. (1995) A time-continuous Markov chain interest model with applications to insurance. Applied Stochastic Models and Data Analysis, 11, 245-256.

[6] Norberg, R. (1997) Discussion of "Stochastic analysis of the interaction between investment and insurance risks". North American Actuarial Journal, 1, 75-76.

[7] Rolski, T., Schmidli, H., Schmidt, V., and Teugels, J. L. (1999) Stochastic Processes for Insurance and Finance. John Wiley, Chichester. 
[8] Ross, S. (1996) Stochastic Processes, 2nd edition. John Wiley, New York.

[9] Shaked, M. and Shanthikumar, J. (1994) Stochastic Orders and their Applications. Academic Press, San Diego.

[10] Sundt, B. and Teugels, J. L. (1995) Ruin estimates under interest force. Insurance: Math. Econom., 16, 7-22.

[11] Sundt, B. and Teugels, J. L. (1997) The adjustment function in ruin estimates under interest force. Insurance: Math. Econom., 19, 85-94.

[12] Willmot, G.E., Cai, J., and Lin, X.S. (2001) Lundberg inequalities for renewal equations. Advances in Applied Probability, 33, 674-689.

[13] Willmot, G.E. and Lin, X.S. (2001) Lundberg Approximations for Compound Distributions with Insurance Applications. Springer-Verlag, New York.

[14] Yang, H. (1999) Non-exponential bounds for ruin probability with interest effect included. Scand. Actuarial J., 99, 66-79. 
Table 1: Upper bounds by the inductive approach

\begin{tabular}{|c|c|c|c|}
\hline$u$ & $\phi\left(u, i_{1}\right)$ & $\varphi\left(u, i_{1}\right)$ & $e^{-R u}$ \\
\hline 0 & 0.8401 & 0.9077 & 1.0000 \\
5 & 0.3806 & 0.5642 & 0.6438 \\
10 & 0.1724 & 0.3507 & 0.4145 \\
15 & 0.0781 & 0.2180 & 0.2669 \\
20 & 0.0354 & 0.1355 & 0.1718 \\
25 & 0.0160 & 0.0842 & 0.1106 \\
30 & 0.0073 & 0.0523 & 0.0712 \\
\hline
\end{tabular}

Table 2: Upper bounds by the martingale approach

\begin{tabular}{|c|c|c|c|}
\hline$u$ & $e^{-R_{2} u}$ & $e^{-R_{3} u}$ & $e^{-R u}$ \\
\hline 0 & 1.0000 & 1.0000 & 1.0000 \\
5 & 0.4545 & 0.6227 & 0.6438 \\
10 & 0.2065 & 0.3877 & 0.4145 \\
15 & 0.0939 & 0.2414 & 0.2669 \\
20 & 0.0427 & 0.1503 & 0.1718 \\
25 & 0.0194 & 0.0936 & 0.1106 \\
30 & 0.0088 & 0.0583 & 0.0712 \\
\hline
\end{tabular}




\section{RECENT ADDITIONS TO THE RESEARCH PAPER SERIES}

No.

Date

84

85

86

87

88

89

90

91

92

93

94

95

96

97

98
FEBRUARY 2001

FEBRUARY 2001

JUNE 2001

SEPTEMBER 2001

NOVEMBER 2001

NOVEMBER 2001

NOVEMBER 2001

NOVEMBER 2001

JANUARY 2002

JANUARY 2002

JANUARY 2002

JANUARY 2002

MAY 2002

JUNE 2002

JUNE 2002
Subject

Author

DISCRETE TIME RISK MODELS UNDER STOCHASTIC FORCES OF INTEREST

MODERN LANDMARKS IN ACTUARIAL SCIENCE Inaugural Professorial Address

LUNDBERG INEQUALITIES FOR RENEWAL EQUATIONS

VOLATILITY, BETA AND RETURN

WAS THERE EVER A MEANINGFUL RELATIONSHIP?

EXPLICIT, FINITE TIME RUIN PROBABILITIES FOR DISCRETE, DEPENDENT CLAIMS

ON THE DISTRIBUTION OF THE DEFICIT AT RUIN WHEN CLAIMS ARE PHASE-TYPE

THE INTEGRATED SQUARE-ROOT PROCESS

ON THE EXPECTED DISCOUNTED PENALTY FUNCTION AT RUIN OF A SURPLUS PROCESS WITH INTEREST

CHAIN LADDER BIAS

FURTHER OBSERVATIONS ON CHAIN LADDER BIAS

A GENERAL CLASS OF RISK MODELS

THE DISTRIBUTION OF THE TIME TO RUIN IN THE CLASSICAL RISK MODEL

A NOTE ON THE MAXIMUM SEVERITY OF RUIN AND RELATED PROBLEMS

UPPER BOUNDS FOR ULTIMATE RUIN PROBABILITIES IN THE SPARRE ANDERSEN MODEL WITH INTEREST

CONTINUOUS COMPOUNDING, VOLATILITY AND THE EQUITY PREMIUM
Jun Cai

David C M Dickson

Gordon E Willmot Jun Cai

$\mathrm{X}$ Sheldon Lin

Richard Fitzherbert

Zvetan G Ignatov Vladimir K Kaishev Rossen S Krachunov

Steve Drekic David C M Dickson David A Stanford Gordon E Willmot

Daniel Dufresne

Jun Cai

David C M Dickson

Greg Taylor

Greg Taylor

Daniel Dufresne

David C M Dickson Howard R Waters

David C M Dickson

Jun Cai

David C M Dickson

Richard Fitzherbert 
No.

Gordon E Willmot David C M Dickson Steve Drekic

David A Stanford

100 AUGUST 2002

ASIAN AND BASKET ASYMPTOTICS

Daniel Dufresne

101

AUGUST 2002

RUIN PROBABILITIES WITH A MARKOV CHAIN INTEREST MODEL

Jun Cai

David C M Dickson

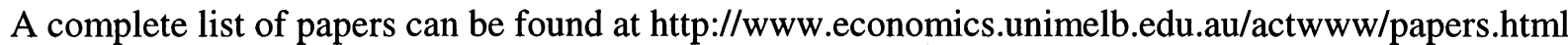




\section{University Library}

\section{- M M I E R R A A gateway to Melbourne's research publications}

Minerva Access is the Institutional Repository of The University of Melbourne

Author/s:

Cai, Jun;Dickson, David C. M.

Title:

Ruin Probabilities with a Markov Chain Interest Model

Date:

2002-08

Citation:

Cai, Jun and Dickson, David C. M. (2002) Ruin Probabilities with a Markov Chain Interest Model.

Persistent Link:

http://hdl.handle.net/11343/33689 